\title{
Analytical Modeling of the Spectral Response of Heterojunction Phototransistors
}

\author{
Hassan A. Khan and Ali A. Rezazadeh
}

\begin{abstract}
Spectral response model for heterojunction phototransistors (HPTs) is developed from resolution of continuity equations that govern the excess optically generated minoritycarrier variation in the active layers of the HPT taking into account the related physical parameters. Realistic boundary conditions have been considered for efficient device operation, and a detailed optical-power absorption profile is constructed for accurate device modeling. The analysis is performed for GaAs-based HPTs, and the measured results at 635,780 , and $850 \mathrm{~nm}$ show a good agreement with theoretical calculations.
\end{abstract}

Index Terms-Heterojunction, modeling, phototransistors, spectral response $(\mathrm{SR})$.

\section{INTRODUCTION}

$\mathbf{S}$ PECTRAL response (SR) is a key performance parameter of heterojunction phototransistors (HPTs) and is critical in their usage in optical applications. The performance of HPTs is supported by their internal current gain (not present in p-i-n or Schottky photodiodes) [1]. Additionally, unlike avalanche photodiodes, HPTs do not suffer from the excessive noise due to an avalanche process [2]. These advantages, along with their process and layer compatibility to heterojunction bipolar transistors, make them highly attractive in the manufacture of monolithic integrated single-chip optical receivers [3], [4].

Modeling of SR for photodiodes has been extensively reported [5]-[7]. In contrast, analytical modeling of the SR for HPTs is rather limited. Chand et al. [8] formulated expressions for flux-dependent collector current, but the boundary conditions chosen for the resolution of continuity equations have been taken from those of p-i-n photodiodes which are highly implausible for efficient operation of HPTs, and no comparison has been made with measured data. Several other attempts at modeling SR require prior knowledge of the device electrical characteristics, such as current gain [9], [10]. In this letter, we have developed the necessary analytical expressions for spectral responsivity of HPTs, and these are compared with the measured results. To the best of our knowledge, this is the first time that such theoretical model, along with a comparison with experimental results, has been reported for HPTs.

Manuscript received July 15, 2009; revised August 7, 2009. First published October 2, 2009; current version published October 23, 2009. The review of this letter was arranged by Editor C. Jagadish.

The authors are with Microwave and Communication Systems Research Group, School of Electrical and Electronic Engineering, The University of Manchester, M60 1QD Manchester, U.K. (e-mail: hassan.khan@postgrad. manchester.ac.uk; ali.rezazadeh@manchester.ac.uk).

Color versions of one or more of the figures in this letter are available online at http://ieeexplore.iee.org.

Digital Object Identifier 10.1109/LED.2009.2030376

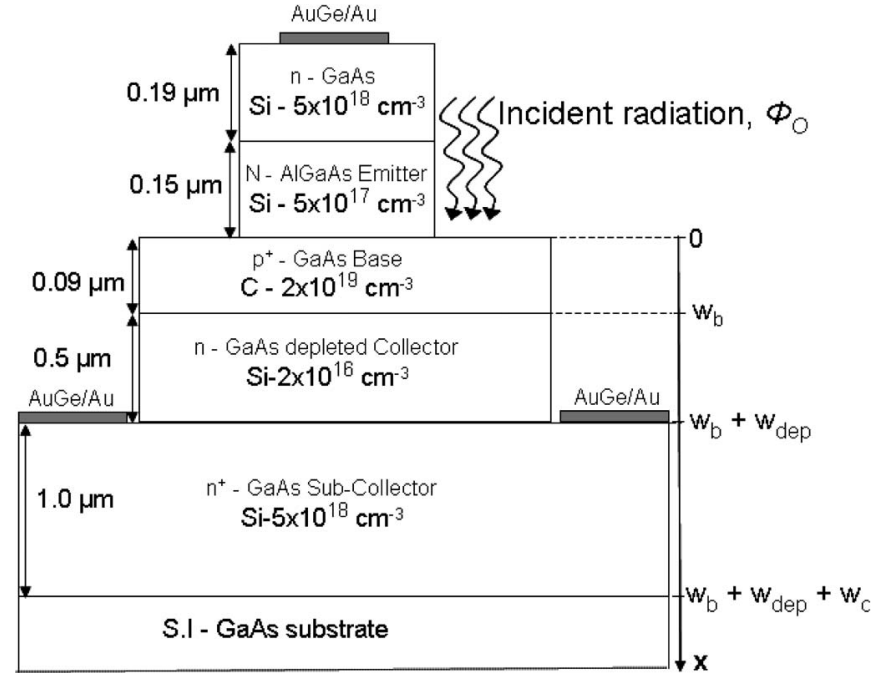

Fig. 1. Schematic device structure of the $\mathrm{Al}_{0.3} \mathrm{Ga}_{0.7} \mathrm{As} / \mathrm{GaAs} \mathrm{HPT}$ showing the incident radiation on the base region.

\section{SR MODELING}

The SR model is based on formulation of semiconductor continuity equations with suitable boundary conditions at the junctions of active device layers. The SR modeling must take into account all of the related device physical parameters. The effect of doping on absorption coefficient at near bandgap wavelengths has been taken into account along with the subtle variations of refractive index caused by the changes in incident photon energies [11], [12]. Auger, radiative, and surface recombinations have also been incorporated in the model. Large circular-geometry (100 $\mu \mathrm{m}$ emitter diameter) HPTs have been fabricated with the device structure shown in Fig. 1. Detailed device fabrication and experimental setup can be found elsewhere [9], [10].

The base surface of the device has been illuminated using an optical fiber, and the incident optical flux is absorbed through the optical window between emitter and subcollector contacts of the device (Fig. 1). The steady-state continuity equations governing the distribution of optically generated minority carriers for low-injection state in the base and subcollector regions are given respectively by

$$
\begin{aligned}
& D_{n} \frac{d^{2} n_{p}}{d x^{2}}-\frac{\left(n_{p}-n_{p o}\right)}{\tau_{n}}+\phi_{b} \alpha_{b} \exp \left(-\alpha_{b} x\right)=0 \\
& D_{p} \frac{d^{2} p_{n}}{d x^{2}}-\frac{\left(p_{n}-p_{n o}\right)}{\tau_{p}}+\phi_{c} \alpha_{c} \exp \left(-\alpha_{c} x\right)=0
\end{aligned}
$$

where $D_{n}\left(D_{p}\right)$ and $\tau_{n}\left(\tau_{p}\right)$ are diffusion coefficient and lifetime for minority carrier electrons (holes), respectively, in $\mathrm{p}^{+}$-GaAs base (n-GaAs subcollector). $n_{p}\left(p_{n}\right)$ and $n_{\mathrm{po}}\left(p_{\text {no }}\right)$ are the total 
and equilibrium electron (hole) density contributions, respectively, in the base (subcollector) region. $\alpha_{b}, \alpha_{c}$ are the optical absorption coefficients for the base and subcollector layers, respectively. $\Phi_{b}$ and $\Phi c$ are the values of incident flux density at the emitter-base $(x=0)$ and collector-subcollector $(x=$ $\left.w_{b}+w_{\text {dep }}\right)$ junctions, respectively. The boundary conditions taken for the base region are modeled by

$$
\begin{gathered}
n_{p}(x=0)=\frac{\phi_{O}\left(1-R_{f}\right)}{s_{n}}=\frac{\phi_{b}}{s_{n}} \\
n_{p}\left(x=w_{b}\right)=\frac{\phi_{b}}{s_{n}} \exp \left(-\alpha_{b} x_{b}\right)+n_{p o}
\end{gathered}
$$

where $\Phi_{o}$ is the incident flux density, $R_{f}$ is the Fresnel reflection coefficient, and $s_{n}$ is the surface-recombination velocity of electrons in the $\mathrm{p}^{+}-\mathrm{GaAs}$ base. The surface recombination will be higher at lower wavelengths as more carriers are generated near the surface. Despite the widely different surface preparation and measurement techniques, the surface-recombination data for GaAs follow the same general trend of proportionality to bulk doping [13]. The recombination due to misfit dislocations at heterostructure interfaces has been minimized by grading the interface. Under these conditions and solving the optically generated minority-carrier density in (1), we have used Fick's law to determine the total electron current at the base-collector junction boundary, and this is given as

$$
\begin{aligned}
I_{n}(\lambda)=- & \frac{q D_{n} A_{E B}}{L_{n} \sinh \frac{w_{b}}{L_{n}}} \\
& \cdot\left\{\frac { \phi _ { b } \alpha _ { b } \tau _ { n } } { \alpha _ { b } ^ { 2 } L _ { n } ^ { 2 } - 1 } \left[\alpha_{b} L_{n} \sinh \left(\frac{w_{b}}{L_{n}}\right) \exp \left(-\alpha_{b} w_{b}\right)\right.\right. \\
& \left.\quad+\cosh \left(\frac{w_{b}}{L_{n}}\right) \exp \left(-\alpha_{b} w_{b}\right)-1\right] \\
& \left.\quad+n_{p}(x=0)\left[\cosh \left(\frac{w_{b}}{L_{n}}\right) \exp \left(-\alpha_{b} w_{b}\right)-1\right]+n_{p o}\right\}
\end{aligned}
$$

where $q$ is the charge of electron, $A_{\mathrm{EB}}$ is the $\mathrm{E}-\mathrm{B}$ junction area, and $L_{n}=D_{n} \tau_{n}$ is the minority-electron diffusion length in the base region. The current due to excess minority holes in the subcollector region can be derived similarly as

$$
\begin{aligned}
I_{p}(\lambda)= & \frac{q D_{p} A_{C}}{L_{p} \sinh \left(\frac{w_{c}}{L_{p}}\right)} \\
& \cdot\left\{\frac { \phi _ { c } \alpha _ { c } \tau _ { p } } { \alpha _ { c } ^ { 2 } L _ { p } ^ { 2 } - 1 } \left[-1+\cosh \left(\frac{w_{c}}{L_{p}}\right) \exp \left(-\alpha_{c} w_{c}\right)\right.\right. \\
& \left.\quad+\sinh \left(\frac{w_{c}}{L_{p}}\right) \alpha_{c} L_{p} \exp \left(-\alpha_{c} w_{c}\right) \alpha_{c} L_{p}\right] \\
& \left.+p_{n}(x=0)\left[\cosh \left(\frac{w_{c}}{L_{p}}\right) \exp \left(-\alpha_{c} w_{c}\right)-1\right]+p_{n o}\right\} .
\end{aligned}
$$

The optical-flux absorption in the depleted collector region

\begin{tabular}{|c|c|c|}
\hline Parameter & Value (respectively) & Reference \\
\hline$D_{n}, D_{p}$ & $50,4 \quad\left[\mathrm{~cm}^{2} . \mathrm{s}^{-1}\right]$ & [12] \\
\hline$\tau_{n}, \tau_{p}$ & $1 \times 10^{-9}, 1.2 \times 10^{-9} \quad[\mathrm{~s}]$ & {$[14,15]$} \\
\hline $\mathrm{L}_{\mathrm{n}}=\left(\tau_{\mathrm{n}} \mathrm{D}_{\mathrm{n}}\right)^{1 / 2}, \mathrm{~L}_{\mathrm{p}}=\left(\tau_{\mathrm{p}} \mathrm{D}_{\mathrm{p}}\right)^{1 / 2}$ & $2.2 \times 10^{-4}, 0.7 \times 10^{-4}[\mathrm{~cm}]$ & [15] \\
\hline 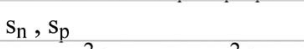 & $3 \times 10^{4}, \sim 10^{6} \quad\left[\mathrm{~cm} . \mathrm{s}^{-1}\right]$ & {$[13,16]$} \\
\hline $\mathrm{n}_{\mathrm{po}}=\mathrm{n}_{\mathrm{i}}^{2} / \mathrm{N}_{\mathrm{A}}, \mathrm{n}_{\mathrm{po}}=\mathrm{n}_{\mathrm{i}}^{2} / \mathrm{N}_{\mathrm{D}}$ & $2.2 \times 10^{-7}, 8.8 \times 10^{-7}\left[\mathrm{~cm}^{-3}\right]$ & [16] \\
\hline$\alpha_{b}(635 \mathrm{~nm}), \alpha_{b}(850 \mathrm{~nm})$ & $3.4 \times 10^{4}, 0.6 \times 10^{4}\left[\mathrm{~cm}^{-1}\right]$ & {$[11,12]$} \\
\hline$\alpha_{\mathrm{dep}}(635 \mathrm{~nm}), \alpha_{\mathrm{dep}}(850 \mathrm{~nm})$ & $3.4 \times 10^{4}, 0.9 \times 10^{4}\left[\mathrm{~cm}^{-1}\right]$ & {$[11,12]$} \\
\hline$\alpha_{c}(635 \mathrm{~nm}), \alpha_{c}(850$ & $3.4 \times 10^{4}, 0.1 \times 10^{4} \quad\left[\mathrm{~cm}^{-1}\right]$ & {$[11,12]$} \\
\hline
\end{tabular}
gives rise to photogenerated current $\left(I_{\mathrm{ph}}\right)$ modeled by [8]

$$
\begin{aligned}
& I_{p h}(\lambda)=-q A_{C} \int_{0}^{w_{\mathrm{dep}}} \Phi_{\mathrm{dep}} \alpha_{\mathrm{dep}} \exp \left(-\alpha_{\mathrm{dep}} x\right) d x \\
& I_{p h}(\lambda)=-q A_{C} \Phi_{\mathrm{dep}}\left[1-\exp \left(-\alpha_{\mathrm{dep}} w_{\mathrm{dep}}\right)\right]
\end{aligned}
$$

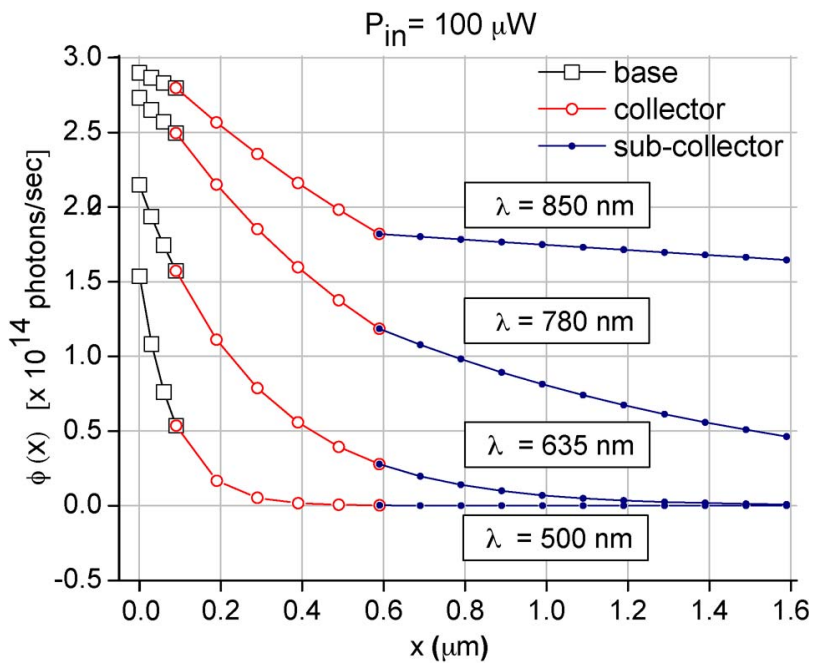

Fig. 2. Optical-flux absorption profile for the $\mathrm{Al}_{0.3} \mathrm{Ga}_{0.7} \mathrm{As} / \mathrm{GaAs} \mathrm{HPT}$ at various incident wavelengths for incident power of $100 \mu \mathrm{W}$.

TABLE I

Material Parameters USED FOR Simulation

where $\Phi_{\text {dep }}$ is the input optical-flux density at the edge of B-C depletion region, $\alpha_{\text {dep }}$ is the absorption coefficient in the depletion region and $A_{C}$ is the area of depleted collector layer. The responsivity $R(\lambda)$ is the ratio of photogenerated current to the input optical power and is given by

$$
R(\lambda)=\lambda \frac{I_{n}(\lambda)+I_{p}(\lambda)+I_{p h}(\lambda)}{h c \Phi_{O}\left(1-R_{f}\right)} .
$$

\section{RESUlts AND DisCUSSION}

Optical-flux absorption profiles, modeled for the $\mathrm{Al}_{0.3} \mathrm{Ga}_{0.7} \mathrm{As} / \mathrm{GaAs} \mathrm{HPT}$ at $500,635,780$, and $850 \mathrm{~nm}$, are shown in Fig. 2. The values of the flux density, used in (5) and (6), at the base and collector of the device can be ascertained from this figure. At $850 \mathrm{~nm}$, the absorption rate in the base, $\mathrm{B}-\mathrm{C}$ depletion region, and subcollector is rather different due to the variation of absorption coefficient with doping in these three layers. However, the absorption coefficient is indifferent for the doping at 635 and $500 \mathrm{~nm}$, and this has been modeled by a single exponential [12]. At $780 \mathrm{~nm}$, the response tends to shift from a single exponential to layer-dependent absorption as the variation in absorption coefficient is minute. A summary of the material parameters used for simulation is given in Table I.

Modeled SR, using (8), along with the measured results at 635,780 , and $850 \mathrm{~nm}$ are shown in Fig. 3. There is no 


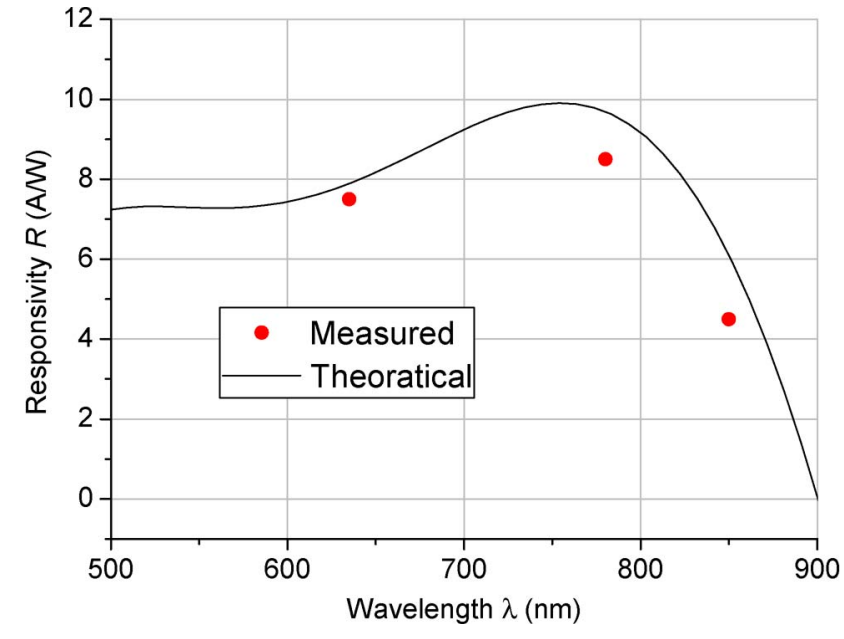

Fig. 3. Measured and calculated SR of the $\mathrm{Al}_{0.3} \mathrm{Ga}_{0.7} \mathrm{As} / \mathrm{GaAs} \mathrm{HPT}$.

measured data at $500 \mathrm{~nm}$ due to unavailability of laser diode at this wavelength at the time of measurement. The responsivity maximizes at around $760 \mathrm{~nm}$ and tends to go down with increasing wavelengths due to a drop in the absorption coefficient. The analytical model described here directly highlights the critical device and material properties affecting the SR of HTPs.

\section{CONCLUSION}

Closed-form analytical expressions for the SR of HPTs have been developed in this letter. Accurate boundary conditions, based on excess minority-carrier continuity equations for active regions of a phototransistor, have been described for the theoretical model. In order to achieve accurate SR modeling, variations in the absorption coefficient with change in the bandgap (due to doping) should be incorporated in the opticalpower absorption profile. The analytical expressions and the critical analysis of several important parameters for HPTs presented here can be utilized for significant performance enhancement through device optimization in photoreceivers employing integrated circuits.

\section{REFERENCES}

[1] S. Chandrasekhar, M. K. Hoppe, A. G. Dentai, C. H. Joyner, and G. J. Qua, "Demonstration of enhanced performance of an InP/InGaAs heterojunction phototransistor with a base terminal," IEEE Electron Device Lett., vol. 12, no. 10, pp. 550-552, Oct. 1991.

[2] P. Chakrabarti, N. K. Agrawal, P. Kalra, S. Agrawal, and G. Gupta, "Noise modeling of an InP/InGaAs heterojunction bipolar phototransistor," Opt. Eng., vol. 42, no. 4, pp. 939-947, Apr. 2003.

[3] A. Leven, V. Houtsma, R. Kopf, Y. Baeyens, and Y.-K. Chen, "InP-based double-heterostructure phototransistors with $135 \mathrm{GHz}$ optical-gain cutoff frequency," Electron. Lett., vol. 40, no. 13, pp. 833-834, Jun. 2004.

[4] H. Kamitsuna, "Ultra-wideband monolithic photoreceivers using HBTcompatible HPTs with novel base circuits, and simultaneously integrated with an HBT amplifier," J. Lightw. Technol., vol. 13, no. 12, pp. 23012307, Dec. 1995.

[5] A. Bouhdada, R. Marrakh, F. Vigue, and J.-P. Faurie, "Modeling of the spectral response of PIN photodetectors impact of exposed zone thickness, surface recombination velocity and trap concentration," Microelectron. Reliab., vol. 44, no. 2, pp. 223-228, Feb. 2004.

[6] H. Zheng, K. Yong, T. Ying-Wen, L. Xue, and F. Jia-Xiong, "Study on the spectral response of the Schottky photodetector of GaN," Chin. Phys., vol. 15, no. 6, pp. 1325-1329, Jun. 2006.

[7] J. A. Gonzalez-Cuevas, T. F. Refaat, M. N. Abedin, and H. E. Elsayed-Ali, "Modeling of the temperature-dependent spectral response of $\operatorname{In}_{1-x} \mathrm{Ga}_{x} \mathrm{Sb}$ infrared photodetectors," Opt. Eng., vol. 45, no. 4, p. 044 001, May 2006.

[8] N. Chand, P. A. Houston, and P. N. Robson, "Gain of a heterojunction bipolar phototransistor," IEEE Trans. Electron Devices, vol. ED-32, no. 3, pp. 622-627, Mar. 1985.

[9] S. A. Bashar and A. A. Rezazadeh, "Fabrication and spectral response analysis of AlGaAs/GaAs and InP/InGaAs HPTs with transparent ITO emitter contacts," Proc. Inst. Elect. Eng.-Optoelectron., vol. 143, no. 1, pp. 89-93, Feb. 1996.

[10] H. A. Khan, A. A. Rezazadeh, and S. C. Subramanian, "Spectral response modelling of heterojunction phototransistors for short wavelength transmission," in Proc. Eur. Microw. Conf. Week, Amsterdam, The Netherlands, Oct. 27-28, 2008, pp. 346-349.

[11] H. C. J. Casey, D. D. Sell, and K. W. Wecht, "Concentration dependence of the absorption coefficient for $\mathrm{n}$ - and p-type GaAs between 1.3 and 1.6 eV," J. Appl. Phys., vol. 46, no. 1, pp. 250-257, Jan. 1975.

[12] "Properties of GaAs," in EMIS Data Review Series, 2nd ed: INSPEC Publ., Brisbane, Australia, 1990 pp. 513-528 .

[13] D. E. Aspnes, "Recombination at semiconductor surfaces and interfaces," in Proc. 2nd Trieste ICTP-IUPAP Semicond. Symp. Surf. Interfaces, Phys. Electron., The Netherlands, Aug. 30-Sep. 3, 1982.

[14] C. J. Hwang, "Doping dependence of hole lifetime in n-type GaAs," J. Appl. Phys., vol. 42, no. 11, pp. 4408-4413, Oct. 1971.

[15] S. M. Sze, Physics of Semiconductor Devices, 2nd ed. New York: Wiley, 2001.

[16] G. A. Acket, W. Nijman, and H. T. Lam, "Electron lifetime and diffusion constant in germanium-doped gallium arsenide," J. Appl. Phys., vol. 45, no. 7, pp. 3033-3040, Jul. 1974. 